\title{
CHARACTERIZATION OF SOME MATRIX CLASSES INVOLVING SOME SETS WITH SPEED
}

\author{
S. DAS AND H. DUTTA
}

Received 11 January, 2018

\begin{abstract}
The paper introduces the notions of boundedness and convergence with speed for difference sequences, and characterizes certain matrix classes associating the sets of such classes of sequences involving the operator $\Delta$ and two speeds $\lambda=\left(\lambda_{k}\right)$ and $\mu=\left(\mu_{k}\right)\left(0<\lambda_{k} \nearrow \infty, 0<\right.$ $\left.\mu_{k} \nearrow \infty\right)$. The results obtained in this paper should easily extendible to difference sequences of higher orders, and even, in combination with multipliers.
\end{abstract}

2010 Mathematics Subject Classification: 46A35, 46A45, 40A05, 40C05

Keywords: Matrix classes, boundedness with speed, convergent with speed, $\Delta$-boundedness, $\Delta$ convergent

\section{INTRODUCTION}

While studying the convergent process, it is important to know the speed of convergence of this process. For example, in the theory of approximation, and using numerical methods for solving differential and integral equations, several methods have been worked out for estimating the speed of convergence.

Let, as usual, $m, c, c_{0}$ be respectively the spaces of all bounded sequences, of all convergent sequences, of all sequences converging to 0 . Throughout this paper indices and summation indices run from 0 to $\infty$ unless otherwise specified.

Let $X, Y$ be two sequence spaces and $A=\left(a_{n k}\right)$ be an infinite matrix with real and complex entries. If for each $x=\left(\xi_{k}\right) \in X$ the series

$$
(A x)_{n}=\sum_{k} a_{n k} \xi_{k}
$$

converges and the sequence $A x=\left\{(A x)_{n}\right\}$ belongs to $Y$, we say that the matrix $A$ transforms $X$ into $Y$. By $(X, Y)$, we denote the set of all matrices which transform

The first author is supported DST-INSPIRE Fellowship, Ministry of Science and Technology, Government of India, Grant No. IF170072. 
$X$ into $Y$.

A matrix $A$ is said to be regular if $A \in(c, c)$ and $\lim _{n}(A x)_{n}=\lim _{k} \xi_{k}$, for each $x=\left(\xi_{k}\right) \in c$, or in short, we write $A \in(c, c ; P)$, where $P$ denotes the preservation of limit. with

Following Kangro ([5], [6], [7]) (also see [2]), a convergent sequence $x=\left(\xi_{k}\right)$

$$
\lim _{k} \xi_{k}=\xi \text { and } v_{k}=\lambda_{k}\left(\xi_{k}-\xi\right)
$$

is called bounded with speed $\lambda$ (shortly, $\lambda$-bounded) if $v_{k}=O(1)$ and convergent with speed $\lambda$ (shortly, $\lambda$-convergent) if the $\operatorname{limit} \lim _{k} v_{k}$ exists and is finite.

The set of all $\lambda$-bounded sequences is denoted by $m^{\lambda}$, and the set of all $\lambda$-convergent sequences by $c^{\lambda}$. It is not difficult to see that $c^{\lambda} \subseteq m^{\lambda} \subseteq c$. In addition to it, for an unbounded sequence $\lambda$ this inclusion is strict. For $\lambda_{k}=O(1)$, we get $c^{\lambda}=m^{\lambda}=c$.

The necessary and sufficient conditions for $A \in\left(m^{\lambda}, m^{\mu}\right), A \in\left(c^{\lambda}, c^{\mu}\right)$, and $A \in$ $\left(c^{\lambda}, m^{\mu}\right)$, were first introduced by Kangro ([5], [6], [7]). The estimation and the comparison of speeds of convergence of series and sequences, based on Kangro's concepts of convergence, boundedness, and summability with speed, have also been studied by Šeletski and Tali ([9], [10]), Stadtmüller and Tali ([11]), and Tammeraid ([12], [13], [14],[15]). For more results on matrix transforms of $m^{\lambda}$ and $c^{\lambda}$, one can refer to ([5], [7], [8]). An improvement of the $\lambda$-convergence has been studied in ([1]).

In this paper, we shall use the notation $\Delta x$ for the sequence of forward differences:

$$
\Delta x_{k}=x_{k}-x_{k+1}, k \in N \text {. }
$$

A sequence $x=\left(\xi_{k}\right)$ is called $\Delta$-convergent if the $\operatorname{limit} \lim _{k} \Delta \xi_{k}$ exists and is finite.

A $\Delta$-convergent sequence $x=\left(\xi_{k}\right)$ with

$$
\lim _{k} \Delta \xi_{k}=\varsigma \text { and } v_{k}=\lambda_{k}\left(\Delta \xi_{k}-\varsigma\right)
$$

is called $\Delta$-bounded with speed $\lambda$ (shorty, $\lambda-\Delta$-bounded) if $v_{k}=O(1)$ and $\Delta$-convergent with speed $\lambda$ (shortly, $\lambda$ - $\Delta$-convergent) if the $\operatorname{limit}_{\lim } v_{k}$ exists and is finite.

By $m_{\Delta}$ and $c_{\Delta}$, we denote the sets of all $\Delta$-bounded sequences and of all $\Delta$ convergent sequences respectively.

The set of all $\lambda$ - $\Delta$-bounded sequences is denoted by $m_{\Delta}^{\lambda}$ and the set of all $\lambda$ - $\Delta$ convergent sequences by $c_{\Delta}^{\lambda}$. It is not difficult to see that $c_{\Delta}^{\lambda} \subseteq m_{\Delta}^{\lambda} \subseteq c_{\Delta}$. In addition to it, for an unbounded sequence $\lambda$ this inclusion is strict. For $\lambda_{k}=O(1)$, we get 
$c_{\Delta}^{\lambda}=m_{\Delta}^{\lambda}=c_{\Delta}$

It is easy to see that every convergent sequence is $\Delta$-convergent, but the converse may not be true. For this, let us consider the following example

Let $x=\left(\xi_{k}\right)=(\kappa)$. Then $\Delta \xi_{k}=\xi_{k}-\xi_{k+1}=-1$. Thus $\left(\xi_{k}\right)$ is divergent but $\Delta$ convergent.

\section{CHARACTERIZATION OF THE MATRIX ClASSES}

We begin this section with few known results that will be required to proof the main results of this paper. Let $e=(1,1, \ldots), e_{k}=(0, \ldots, 0,1,0, \ldots)$, where 1 is in the $(k+1)^{t h}$ - position, and $\lambda^{-1}=\left(\frac{1}{\lambda_{k}}\right)$.

Theorem 1 ([3], [4], Silverman-Toeplitz). $A=\left(a_{n k}\right)$ is regular, i.e., $A \in(c, c ; P)$, if and only if

$$
\begin{gathered}
\sup _{n \geq 0} \sum_{k}\left|a_{n k}\right|<\infty \\
\lim _{n} a_{n k}=\delta_{k}
\end{gathered}
$$

and

$$
\lim _{n} \sum_{k} a_{n k}=\delta
$$

with $\delta_{k} \equiv 0$ and $\delta \equiv 1$

Theorem 2 ([3], [4]). Let $A=\left(a_{n k}\right)$ be a matrix method. Then $A \in(c, c)$ if and only if (2.1) holds and the finite limits $\delta_{k}$ and $\delta$ exist.

Theorem 3 ([3], [4]). A method $A=\left(a_{n k}\right) \in\left(c_{0}, c\right)$ if and only if conditions (2.1) and (2.2) hold.

Theorem 4 ([3], [4]). Let $A=\left(a_{n k}\right)$ be a matrix method. Then $A \in(m, m)=$ $(c, m)=\left(c_{0}, m\right)$ if and only if condition (2.1) holds.

Theorem 5 ([3], [4]). A method $A=\left(a_{n k}\right) \in(m, c)$ if and only if conditions (2.1) and (2.2) are satisfied and $\lim _{n} \sum_{k}\left|a_{n k}-\delta_{k}\right|=0$.

In this case

$$
\lim _{n}(A x)_{n}=\sum_{k} \delta_{k} \xi_{k}
$$

for every $x=\left(\xi_{k}\right) \in m$.

Theorem 6. A method $A=\left(a_{n k}\right) \in\left(m^{\lambda}, m_{\Delta}^{\mu}\right)$ if and only if

$$
\begin{gathered}
\lim _{n} \Delta a_{n k}=\delta_{k}^{l}, \\
\text { Ae } \in m_{\Delta}^{\mu},
\end{gathered}
$$




$$
\begin{gathered}
\sum_{k} \frac{\left|\Delta a_{n k}\right|}{\lambda_{k}}=O(1), \\
\mu_{n} \sum_{k} \frac{\left|\Delta a_{n k}-\delta_{k}^{/}\right|}{\lambda_{k}}=O(1) .
\end{gathered}
$$

If $\mu_{n}=O(1)$ and $\lambda_{n} \neq O(1)$, then in (2.7), it is necessary to replace $O$ (1) by $o(1)$.

Proof. Necessity: Suppose that $A=\left(a_{n k}\right) \in\left(m^{\lambda}, m_{\Delta}^{\mu}\right)$. It is obvious that $e_{k}, e \in$ $m^{\lambda}$. Hence conditions (2.4) and (2.5) are fulfilled.

Let $x=\left(\xi_{k}\right) \in m^{\lambda}$, then from (1.1) we have

$\xi_{k}=\frac{v_{k}}{\lambda_{k}}+\xi$ where $\lim _{k} \xi_{k}=\xi, v_{k}=O(1)$ it follows that

$$
(A x)_{n}=\sum_{k} \frac{a_{n k}}{\lambda_{k}} v_{k}+\xi \sum_{k} a_{n k}
$$

and

$$
\begin{aligned}
\Delta(A x)_{n} & =(A x)_{n}-(A x)_{n+1} \\
& =\sum_{k} \frac{a_{n k}}{\lambda_{k}} v_{k}+\xi \sum_{k} a_{n k}-\sum_{k} \frac{a_{(n+1) k}}{\lambda_{k}} v_{k}-\xi \sum_{k} a_{(n+1) k} \\
& =\sum_{k} \frac{\left(a_{n k}-a_{(n+1) k}\right)}{\lambda_{k}} v_{k}+\xi \sum_{k}\left(a_{n k}-a_{(n+1) k}\right) \\
& =\sum_{k} \frac{\Delta a_{n k}}{\lambda_{k}} v_{k}+\xi \sum_{k} \Delta a_{n k} .
\end{aligned}
$$

As $\left(\sum_{k} a_{n k}\right) \in m_{\Delta}^{\mu}$, by (2.5), then from (2.8) we can assert that the method $A_{\lambda}=\left(\frac{\Delta a_{n k}}{\lambda_{k}}\right)$ transforms the bounded sequence $\left(v_{k}\right)$ into $c$.

Now we assume that $\lambda_{n} \neq O(1)$. Then for every sequence $\left(v_{k}\right) \in m$, the sequence $\left(\frac{v_{k}}{\lambda_{k}}\right) \in c_{0}$. But for $\left(\frac{v_{k}}{\lambda_{k}}\right)$, there exists a convergent sequence $x=\left(\xi_{k}\right)$ such that $\lim _{k} \xi_{k}=\xi$ and $\frac{v_{k}}{\lambda_{k}}=\left(\xi_{k}-\xi\right)$. Thus, for every sequence $\left(v_{k}\right) \in m$, there exists a sequence $\left(\xi_{k}\right) \in m^{\lambda}$ such that $v_{k}=\lambda_{k}\left(\xi_{k}-\xi\right)$. Hence $A_{\lambda} \in(m, c)$. This implies by Theorem 5, the condition (2.6) holds,

$$
\lim _{n} \sum_{k} \frac{\left|\Delta a_{n k}-\delta_{k}^{\prime}\right|}{\lambda_{\kappa}}=0
$$

and $\phi^{\prime}=\lim _{n} \Delta(A x)_{n}=\sum_{k} \frac{\delta_{k}^{\prime}}{\lambda_{k}} v_{k}+\xi \lim _{n} \sum_{k} \Delta a_{n k}$. 
If $\mu_{n} \neq O(1)$, then writing

$$
\begin{aligned}
& \mu_{n}\left(\Delta(A x)_{n}-\phi^{\prime}\right)= \\
& \quad=\mu_{n} \sum_{k} \frac{\Delta a_{n k-} \delta_{k}^{l}}{\lambda_{k}} v_{k}+\xi \mu_{n}\left(\sum_{k} \Delta a_{n k}-\lim _{n} \sum_{k} \Delta a_{n k}\right) .
\end{aligned}
$$

By (2.5) we can conclude that the method

$$
A_{\lambda, \mu_{\Delta}}=\left(\mu_{n} \frac{\Delta a_{n k-} \delta_{k}^{l}}{\lambda_{k}}\right) \in(m, m) .
$$

This implies by Theorem 4, condition (2.7) is fulfilled.

If $\mu_{n}=O(1)$, then in (2.7) it is necessary to replace $O(1)$ by $o(1)$; which is similar to $(2.9)$.

If $\lambda_{n}=O(1)$, then the proof is similar to the case $\lambda_{n} \neq O(1)$, but in this case $v_{k}=o(1)$, and instead of the Theorem 5, it is necessary to use the Theorem 3 .

Sufficiency: Conversely assume that the conditions (2.4)-(2.7) are valid. Also, for every $x=\left(\xi_{k}\right) \in m^{\lambda}$, the relation (2.8) holds and by (2.5), $\left(\sum_{k} a_{n k}\right) \in m_{\Delta}^{\mu}$. If $\lambda_{n} \neq O(1)$ and $\mu_{n}=O(1)$, then using Theorem 5, we can conclude that the method $A_{\lambda} \in(m, c)$ by (2.4), (2.6) and (2.9) (in this case, we have (2.9) instead of (2.7)). Thus $A \in\left(m^{\lambda}, c_{\Delta}\right)$

If $\lambda_{n} \neq O(1)$ and $\mu_{n} \neq O(1)$, then validity of (2.9) follows from the validity of (2.7). In this case also $A_{\lambda} \in(m, c)$ by (2.4), (2.6) and (2.9), that is $A \in\left(m^{\lambda}, c_{\Delta}\right)$. Therefore, we can assert that the limit $\phi^{\prime}$ exists and is finite and therefore relation (2.10) is fulfilled for every $x=\left(\xi_{k}\right) \in m^{\lambda}$. Hence by (2.7) and using Theorem 4, we have $A_{\lambda, \mu_{\Delta}} \in(m, m)$ and by (2.5), we have $A \in\left(m^{\lambda}, m_{\Delta}^{\mu}\right)$. For $\lambda_{n}=O(1)$, the proof is obvious.

Theorem 7. A method $A=\left(a_{n k}\right) \in\left(c^{\lambda}, c_{\Delta}^{\mu}\right)$ if and only if conditions (2.6) and (2.7) are fulfilled and

$$
\begin{gathered}
A e_{k} \in c_{\Delta}^{\mu}, \\
A e \in c_{\Delta}^{\mu}, \\
A \lambda^{-1} \in c_{\Delta}^{\mu} .
\end{gathered}
$$


If $A \in\left(c^{\lambda}, c_{\Delta}^{\mu}\right)$, then

$$
\begin{aligned}
\lim _{n} \mu_{n}\left(\Delta(A x)_{n}-\phi^{\prime}\right)= & \sum_{k} a_{k}^{\lambda, \mu_{\Delta}}\left(v_{k}-v\right)+\lim _{n} \mu_{n}\left(\sum_{k} \Delta a_{n k}-\delta^{\prime}\right) \xi \\
& +\lim _{n} \mu_{n}\left(\sum_{k} \frac{\Delta a_{n k}}{\lambda_{k}}-a^{\lambda}\right) v,
\end{aligned}
$$

where

$$
\phi^{\prime}=\lim _{n} \Delta(A x)_{n}, v=\lim _{k} v_{k}
$$

and

$$
\begin{gathered}
\delta^{\prime}=\lim _{n} \sum_{k} \Delta a_{n k}, \delta_{k}^{l}=\lim _{n} \Delta a_{n k}, \\
a^{\lambda}=\lim _{n} \sum_{k} \frac{\Delta a_{n k}}{\lambda_{k}}, a_{k}^{\lambda, \mu_{\Delta}}=\lim _{n} \mu_{n} \frac{\Delta a_{n k}-\delta_{k}^{\prime}}{\lambda_{k}}
\end{gathered}
$$

Proof. Necessity: Suppose that $A \in\left(c^{\lambda}, c_{\Delta}^{\mu}\right)$. It is not difficult to see that $e_{k}, e$, $\lambda^{-1} \in c^{\lambda}$ and so the conditions (2.11)-(2.13) hold. For every $x=\left(\xi_{k}\right) \in c^{\lambda}$, the equality (2.8) is satisfied and by (2.12) the limit $\delta /$ exists, so the method $A_{\lambda}$ transforms the convergent sequence $\left(v_{k}\right)$ into $c$. Similar to the proof of necessary part of Theorem 6 , it can be easily shown that, for every sequence $\left(v_{k}\right) \in c$, there exists a sequence $x=\left(\xi_{k}\right) \in c^{\lambda}$ such that $v_{k}=\lambda_{k}\left(\xi_{k}-\xi\right)$. Hence $A_{\lambda} \in(c, c)$. This means that the finite limits $\delta_{k}^{l}$ and $a^{\lambda}$ exist and condition (2.6) is fulfilled by virtue of Theorem 2 . Using relation (2.8), for every $x \in c^{\lambda}$, we can write

$$
\phi^{\prime}=\lim _{n} \Delta(A x)_{n}=a^{\lambda} v+\sum_{k} \frac{\delta_{k}^{\prime}}{\lambda_{k}}\left(v_{k}-v\right)+\xi \delta^{\prime},
$$

where $\xi=\lim _{k} \xi_{k}$ and $v=\lim _{k} v_{k}$.

Now using relations (2.8) and (2.15), we get

$$
\begin{aligned}
\mu_{n}\left(\Delta(A x)_{n}-\phi^{\prime}\right)= & \mu_{n} \sum_{k} \frac{\Delta a_{n k}-\delta_{k}^{\prime}}{\lambda_{k}}\left(v_{k}-v\right)+\mu_{n}\left(\sum_{k} \Delta a_{n k}-\delta^{\prime}\right) \xi \\
& +\mu_{n}\left(\sum_{k} \frac{\Delta a_{n k}}{\lambda_{k}}-a^{\lambda}\right) v .
\end{aligned}
$$

As $n \rightarrow \infty$, the finite limits for the last two summands in the right hand side of (2.16) exist by conditions (2.12) and (2.13). This implies that the method $A_{\lambda, \mu_{\Delta}} \in\left(c_{0}, c\right)$. Thus using Theorem 3, the condition (2.7) is satisfied. Lastly, relation (2.14) holds from (2.16). 
Sufficiency: Suppose that (2.6)-(2.7) and (2.11)-(2.13) are fulfilled. We observe that the relation (2.16) holds for every $x=\left(\xi_{k}\right) \in c^{\lambda}$ and also the finite limits $\delta_{k}^{\prime}, \delta^{\prime}, a^{\lambda}$ exist by (2.11), (2.12) and (2.13) respectively. Since (2.6) also holds, so $A_{\lambda} \in(c, c)$ by Theorem 2 and therefore for every $x \in c^{\lambda}$, relations (2.15) and (2.16) hold. Now by conditions (2.12) and (2.13), the finite limits for the last two summands in the right side of (2.16) exist as $n \rightarrow \infty$. Finally using conditions (2.7), (2.11) and Theorem 3 we can conclude that the method $A_{\lambda, \mu_{\Delta}} \in\left(c_{0}, c\right)$. Hence, $A \in\left(c^{\lambda}, c_{\Delta}^{\mu}\right)$.

It is easy to see that conditions (2.4) and (2.6) imply the condition

$$
\sum_{k} \frac{\left|\delta_{k}^{/}\right|}{\lambda_{k}}<\infty
$$

Also conditions (2.7) and (2.17) imply condition (2.6). Therefore, from Theorem 6 and Theorem 7, we get the following corollary:

Corollary 1. The condition (2.6) in Theorem 6 and Theorem 7 can be replaced by the condition (2.17).

Using Theorem 6 and Corollary 1, we get the following corollary:

Corollary 2. A method $A=\left(a_{n k}\right) \in\left(m^{\lambda}, c_{\Delta}\right)$ if and only if the conditions (2.4), (2.6) and (2.9) are fulfilled and the finite limit $\lim _{n} \sum_{k} \Delta a_{n k}=\delta^{\prime}$ exists. Also the condition (2.6) can be replaced by the condition (2.17).

Theorem 8. A method $A=\left(a_{n k}\right) \in\left(c^{\lambda}, m_{\Delta}^{\mu}\right)$ if and only if the conditions (2.4)(2.7) are satisfied.

Also if $\mu_{n}=O(1)$ and $\lambda_{n} \neq O(1)$, then in (2.7), it is necessary to replace $O(1)$ by $o(1)$.

Proof. Necessary Part: Suppose that $A=\left(a_{n k}\right) \in\left(c^{\lambda}, m_{\Delta}^{\mu}\right)$. It is easy to see that $e_{k}, e \in c^{\lambda}$. Hence conditions (2.4) and (2.5) are valid. As equality (2.8) holds for every $x=\left(\xi_{k}\right) \in c^{\lambda}$, and $\left(\sum_{k} a_{n k}\right) \in m_{\Delta}^{\mu}$ by (2.5), then the method $A_{\lambda}$ transforms the convergent sequence $\left(v_{k}\right)$ into $c$. Similar to the proof of necessary part of Theorem 6 , it can be easily shown that for every sequence $\left(v_{k}\right) \in c$, there exists a sequence $x=\left(\xi_{k}\right) \in c^{\lambda}$ such that $v_{k}=\lambda_{k}\left(\xi_{k}-\xi\right)$. Hence $A_{\lambda} \in(c, c)$. This implies by Theorem 2, the condition (2.6) is satisfied.

Using condition (2.8), for every $x=\left(\xi_{k}\right) \in c^{\lambda}$, we can write

$$
\phi^{\prime}=\lim _{n} \Delta(A x)_{n}=\sum_{k} \frac{\delta_{k}^{\prime}}{\lambda_{\kappa}} v_{k}+\xi \lim _{n} \sum_{k} \Delta a_{n k} .
$$

If $\mu_{n} \neq O(1)$, then from relation (2.10) and using condition (2.5) we can assert that the method

$$
A_{\lambda, \mu_{\Delta}} \in(c, m)
$$


Therefore using Theorem 4, condition (2.7) is fulfilled.

For $\mu_{n}=O(1)$, then in (2.7) it is necessary to replace $O(1)$ by $o(1)$; which is equivalent to (2.9).

If $\lambda_{n}=O(1)$, then the proof is similar to the case $\lambda_{n} \neq O(1)$, but in this case $\left(v_{k}\right) \in c_{0}$, and instead of the Theorem 2, it is necessary to use the Theorem 3 .

Sufficient Part: It is obvious from the Theorem 6.

\section{REFERENCES}

[1] A. Aasma, "Convergence acceleration and improvement by regular matrices. In: Dutta, H. and Rhoades, B.E. (eds.) Current Topics in Summability Theory and Applications." Springer, Singapore, vol. 1, pp. 141-180, 2016, doi: 10.1007/978-981-10-0913-6_4.

[2] A. Aasma, H. Dutta, and P. Natarajan, An introductory course in summability theory. New York: Wiley, 2017. doi: 10.1002/9781119397786.

[3] J. Boos, Classical and modern methods in summability. Oxford: Oxford University Press, 2000.

[4] G. Hardy, Divergent Series. Oxford: Oxford University Press, 1949.

[5] G. Kangro, "O množitelyah summirujemosti tipa Bora-Hardy dlya zadannoi ckorosti I (On the summability factors of the Bohr-Hardy type for a given speed I)." Eesti NSV Tead. Akad. Toimetised Fü̈is.-Mat., vol. 18, no. 2, pp. 137-146, 1969.

[6] G. Kangro, "O množitelyah summirujemosti tipa Bora-Hardy dlya zadannoi ckorosti II (On the summability factors of the Bohr-Hardy type for a given speed II)." Eesti NSV Tead. Akad. Toimetised Fü̈is.-Mat., vol. 18, no. 4, pp. 387-395, 1969.

[7] G. Kangro, "Množiteli summirujemosti dlya ryadov, $\lambda$-ogranitšennõh metodami Rica i Cezaro (Summability factors for the series $\lambda$-bounded by the methods of Riesz and Cesàro)." Tartu Riikl. ÜI. Toimetised, vol. 277, pp. 136-154, 1971.

[8] T. Leiger, Funktsionaalanalü̈̈si meetodid summeeruvusteooroias (Methods of functional analysis in summability theory). Tartu: Tartu Ülikool, 1992.

[9] A. Šeletski and A. Tali, "Comparison of speeds of convergence in Riesz-Type families of summability methods." Proc. Est. Acad. Sci., vol. 57, no. 1, pp. 70-80, 2008, doi: 10.3176/proc.2008.2.02.

[10] A. Šeletski and A. Tali, "Comparison of speeds of convergence in Riesz-Type families of summability methods II." Math. Model. Anal., vol. 15, no. 1, pp. 103-112, 2011, doi: 10.3846/13926292.2010.15.103-112.

[11] U. Stadtmüller and A. Tali, "Comparison of certain summability methods by speeds of convergence." Anal. Math., vol. 29, no. 3, pp. 227-242, 2003, doi: 10.1023/A:1025419305735.

[12] I. Tammeraid, "Convergence acceleration and linear methods." Math. Model. Anal., vol. 8, no. 1, pp. 87-92, 2003, doi: 10.1080/13926292.2003.9637213.

[13] I. Tammeraid, "Generalized linear methods and convergence acceleration." Math. Model. Anal., vol. 8, no. 4, pp. 329-335, 2003, doi: 10.1080/13926292.2003.9637234.

[14] I. Tammeraid, "Several remarks on acceleration of convergence using generalized linear methods of summability." J. Comput. Appl. Math., vol. 159, no. 2, pp. 365-373, 2003, doi: 10.1016/S03770427(03)00539-9.

[15] I. Tammeraid, "Generalized Riesz method and convergence acceleration." Math. Model. Anal., vol. 9, no. 4, pp. 341-348, 2004, doi: 10.1080/13926292.2004.9637264. 
Authors' addresses

S. Das

Gauhati University, Mathematics Department, Guwahati, 781014, India

E-mail address: dshilpa7660gmail.com

H. Dutta

Gauhati University, Mathematics Department, Guwahati, 781014, India

E-mail address: hemen_dutta08@rediffmail.com 